\title{
EXISTENCE AND GLOBAL ATTRACTIVITY \\ OF THE UNIQUE POSITIVE PERIODIC SOLUTION \\ FOR DISCRETE HEMATOPOIESIS MODEL
}

\author{
ZHIJIAN YAO
}

\begin{abstract}
In this paper, a discrete Hematopoiesis model is studied. By using fixed point theorem of decreasing operator, we obtain sufficient conditions for the existence of unique positive periodic solution. Particularly,we give iterative sequence which converges to the positive periodic solution. In addition, the global attractivity of positive periodic solution is also investigated.
\end{abstract}

\section{Introduction}

Biological dynamic models are very important and hot research topics. In 1977, Mackey and Glass [14] investigated the Hematopoiesis model

$$
x^{\prime}(t)=-a x(t)+\frac{\beta}{1+x^{n}(t-\tau)}
$$

which described the production of blood cells. Gyori and Ladas [7] have investigated the global attractivity of positive equilibrium for the above model (1.1). Moreover, the model (1.1) and some generalized models have been investigated by many authors, see [3], [5], [9]-[11], [15]-[17].

2010 Mathematics Subject Classification. 39A23, 39A30.

Key words and phrases. Discrete Hematopoiesis model, periodic solution, uniqueness, global attractivity, fixed point theorem of decreasing operator.

This work is supported by the Natural Science Foundation of Anhui Province (No. KJ2014A043). 
The assumption that the environment is constant is rarely the case in real life. When the environmental fluctuation is taken into account, a model must be nonautonomous. Due to the various seasonal effects of the environmental factors in real life situation (e.g. seasonal effects of weather, food supplies, mating habits, harvesting, etc.), the effects of periodically varying environment are important, it is rational and practical to study the biological system with periodic coefficients. Many authors [17], [16], [9] have studied nonautonomous differential equations with periodic coefficients of the above model (1.1).

Though most models are described with differential equations, the discretetime models are more appropriate than the continuous ones when the size of the population is rarely small or the population has non-overlapping generations [1]. However, the studies in the past literature [7], [5], [11], [15], [10], [3], [17], [16], [9] were concerned with the continuous case of the above model (1.1). To our knowledge, studies on the uniqueness and global attractivity of positive periodic solution for discrete models are scarce.

Motivated by the above facts, in this paper, we investigate the following Hematopoiesis difference equation

$$
\Delta x(k)=-a(k) x(k)+\frac{b(k)}{1+x^{n}(k-\tau(k))}
$$

where

$$
\begin{gathered}
\Delta x(k)=x(k+1)-x(k), \quad n>0, \\
a(k): Z \rightarrow(0,1), \quad b(k): Z \rightarrow(0,+\infty), \quad \tau(k): Z \rightarrow Z^{+} .
\end{gathered}
$$

$a(k), b(k), \tau(k)$ are $\omega$-periodic functions, i.e.

$$
a(k)=a(k+\omega), \quad b(k)=b(k+\omega), \quad \tau(k)=\tau(k+\omega), \quad \omega \in Z^{+} .
$$

Let $\tau^{*}=\max _{0 \leq k \leq \omega} \tau(k)$, the initial condition of equation (1.2) is $x(k)=\phi(k)>0$ for $-\tau^{*} \leq k \leq 0$.

In the study of biological systems, an important ecological problem concerns the existence of positive periodic solutions. Recently, many authors investigated the existence of positive periodic solution by using Krasnosel'skiu cone fixed point theorem and Mawhin's coincidence degree theory [2], [4], [8], [9], [12], [19]. Most of the literature concerned the existence of at least one positive periodic solution $[2],[4],[8],[9],[12],[13],[19]$.

Few papers study the existence and global attractivity of unique positive periodic solution for discrete models. For the existence and uniqueness of positive periodic solution, the method used in most of the past literature is contraction mapping fixed point theorem and Liapunov functionals.

In this paper, different from the past literature, we aim to obtain sufficient conditions that guarantee the existence of unique positive periodic solution of 
discrete model (1.2) by using fixed point theorem of decreasing operator. Particularly, we give iterative sequence which converges to the positive periodic solution. We also obtain sufficient conditions for the global attractivity of unique positive periodic solution by means of some analysis tools.

\section{Preliminaries}

Definition 2.1. Let $X$ be a Banach space and $P$ be a closed nonempty subset of $X, P$ is called a cone if

(a) $x \in P, \lambda \geq 0$ implies $\lambda x \in P$;

(b) $x \in P,-x \in P$ implies $x=0$.

Every cone $P \subset X$ induces an ordering in $X$, we define " $\leq$ "with respect to $P$ by $x \leq y$ if and only if $y-x \in P$.

Definition 2.2. A cone $P$ of $X$ is called normal cone if there exists a positive constant $\sigma$, such that $\|x+y\| \geq \sigma$ for any $x, y \in P,\|x\|=\|y\|=1$.

Definition 2.3. Let $P$ be a cone of $X$ and $A: P \rightarrow P$ an operator. $A$ is called decreasing if $0 \leq x \leq y$ implies $A x \geq A y$.

The following fixed point theorem of decreasing operator (see [6]) is an important tool in our proofs.

LEMMA 2.4 ([6]). Suppose that:

(a) $P$ is normal cone of Banach space $X$, operator $A: P \rightarrow P$ is decreasing;

(b) $A(0)>0, A^{2}(0) \geq \varepsilon_{0} A(0)$, where $\varepsilon_{0}>0$;

(c) For any $0<a<c<1$, there exists $\eta=\eta(a, c)>0$ such that $A(\lambda x) \leq$ $[\lambda(1+\eta)]^{-1} A x$ for any $a \leq \lambda \leq c$ and $0<x \leq A(0)$.

Then $A$ has a unique positive fixed point $x^{*}>0$. Moreover, $\left\|x_{k}-x^{*}\right\| \rightarrow 0$, $(k \rightarrow \infty)$, where $x_{k}=A x_{k-1},(k=1,2, \ldots)$ for any initial $x_{0} \in P$.

In this paper, we will use the above Lemma 2.4 to investigate the existence of unique positive periodic solution of model (1.2).

REMARK 2.5. In Lemma 2.4, the operator $A$ needs neither continuity nor compactness.

Let $X=\{x(k) \mid x(k)=x(k+\omega)\}$, for $x \in X$, we define $\|x\|=\max _{k \in Z, 0 \leq k \leq \omega}|x(k)|$, then $X$ is Banach space.

Let $\bar{a}=\max _{0 \leq k \leq \omega-1} a(k), \underline{a}=\min _{0 \leq k \leq \omega-1} a(k), \bar{b}=\max _{0 \leq k \leq \omega-1} b(k)$,

$$
m=\frac{\prod_{r=0}^{\omega-1}(1-a(r))}{1-\prod_{r=0}^{\omega-1}(1-a(r))}, \quad M=\frac{1}{1-\prod_{r=0}^{\omega-1}(1-a(r))}, \quad G(k, s)=\frac{\prod_{r=s+1}^{k+\omega-1}(1-a(r))}{1-\prod_{r=0}^{\omega-1}(1-a(r))},
$$


for $k \leq s \leq k+\omega-1$. Then we know $G(k, s)$ is $\omega$-periodic in both variables, and

$$
m=\frac{\prod_{r=0}^{\omega-1}(1-a(r))}{1-\prod_{r=0}^{\omega-1}(1-a(r))} \leq G(k, s) \leq \frac{1}{1-\prod_{r=0}^{\omega-1}(1-a(r))}=M .
$$

LEMMA 2.6. $x(k)$ is the $\omega$-periodic solution of equation (1.2) if and only if $x(k)$ is the $\omega$-periodic solution of the following equation

$$
x(k)=\sum_{s=k}^{k+\omega-1}\left(G(k, s) \frac{b(s)}{1+x^{n}(s-\tau(s))}\right) .
$$

Proof. It is easy to see that equation (1.2) is equivalent to

$$
x(k+1)-(1-a(k)) x(k)=\frac{b(k)}{1+x^{n}(k-\tau(k))}
$$

Multiplying two sides of (2.1) by $\prod_{r=0}^{k} 1 /(1-a(r))$, we get

$$
\Delta\left(x(k) \prod_{r=0}^{k-1} \frac{1}{1-a(r)}\right)=\frac{b(k)}{1+x^{n}(k-\tau(k))} \prod_{r=0}^{k} \frac{1}{1-a(r)}
$$

Summing two sides of (2.2) from $k$ to $k+\omega-1$, we get

$$
\begin{aligned}
x(k+\omega) \prod_{r=0}^{k+\omega-1} \frac{1}{1-a(r)}-x(k) \prod_{r=0}^{k-1} \frac{1}{1-a(r)} \\
=\sum_{s=k}^{k+\omega-1}\left(\frac{b(s)}{1+x^{n}(s-\tau(s))} \prod_{r=0}^{s} \frac{1}{1-a(r)}\right)
\end{aligned}
$$

Since $x(k+\omega)=x(k)$, we have

$$
x(k)\left(\prod_{r=0}^{k+\omega-1} \frac{1}{1-a(r)}-\prod_{r=0}^{k-1} \frac{1}{1-a(r)}\right)=\sum_{s=k}^{k+\omega-1}\left(\frac{b(s)}{1+x^{n}(s-\tau(s))} \prod_{r=0}^{s} \frac{1}{1-a(r)}\right)
$$

That is

$$
\begin{aligned}
x(k) \prod_{r=0}^{k-1} \frac{1}{1-a(r)}\left(\prod_{r=k}^{k+\omega-1} \frac{1}{1-a(r)}\right. & -1) \\
& =\sum_{s=k}^{k+\omega-1}\left(\frac{b(s)}{1+x^{n}(s-\tau(s))} \prod_{r=0}^{s} \frac{1}{1-a(r)}\right)
\end{aligned}
$$


Hence we get

$$
\begin{aligned}
& x(k)=\frac{\prod_{r=0}^{k+\omega-1}(1-a(r))}{1-\prod_{r=k}^{k+\omega-1}(1-a(r))} \sum_{s=k}^{k+\omega-1}\left(\frac{b(s)}{1+x^{n}(s-\tau(s))} \prod_{r=0}^{s} \frac{1}{1-a(r)}\right) \\
& =\frac{\prod_{r=0}^{k+\omega-1}(1-a(r))}{1-\prod_{r=0}^{\omega-1}(1-a(r))} \sum_{s=k}^{k+\omega-1}\left(\frac{b(s)}{1+x^{n}(s-\tau(s))} \prod_{r=0}^{s} \frac{1}{1-a(r)}\right) \\
& =\sum_{s=k}^{k+\omega-1}\left(\frac{\prod_{r=0}^{k+\omega-1}(1-a(r))}{1-\prod_{r=0}^{\omega-1}(1-a(r))} \frac{b(s)}{1+x^{n}(s-\tau(s))} \prod_{r=0}^{s} \frac{1}{1-a(r)}\right) \\
& =\sum_{s=k}^{k+\omega-1}\left(\frac{\prod_{r=s+1}^{k+\omega-1}(1-a(r))}{1-\prod_{r=0}^{\omega-1}(1-a(r))} \frac{b(s)}{1+x^{n}(s-\tau(s))}\right) \\
& =\sum_{s=k}^{k+\omega-1}\left(G(k, s) \frac{b(s)}{1+x^{n}(s-\tau(s))}\right) .
\end{aligned}
$$

The proof is complete.

We define operator $A: X \rightarrow X$,

$$
(A x)(k)=\sum_{s=k}^{k+\omega-1}\left(G(k, s) \frac{b(s)}{1+x^{n}(s-\tau(s))}\right)
$$

Obviously, $x(k) \in X$ is the $\omega$-periodic solution of equation (1.2) if and only if $x$ is the fixed point of operator $A$.

Define cone $\Omega=\{x \mid x \in X, x(k) \geq 0, x(k) \geq \delta\|x\|\}, \delta=m / M$.

Lemma 2.7. $A \Omega \subset \Omega$.

Proof. For any $x \in \Omega$,

$$
\begin{aligned}
\|A x\| & =\max _{k \in Z, 0 \leq k \leq \omega}|(A x)(k)|=\max _{k \in Z, 0 \leq k \leq \omega}\left|\sum_{s=k}^{k+\omega-1}\left(G(k, s) \frac{b(s)}{1+x^{n}(s-\tau(s))}\right)\right| \\
& \leq M \max _{k \in Z, 0 \leq k \leq \omega}\left|\sum_{s=k}^{k+\omega-1}\left(\frac{b(s)}{1+x^{n}(s-\tau(s))}\right)\right|
\end{aligned}
$$




$$
=M \max _{k \in Z, 0 \leq k \leq \omega}\left|\sum_{s=0}^{\omega-1}\left(\frac{b(s)}{1+x^{n}(s-\tau(s))}\right)\right|=M \sum_{s=0}^{\omega-1}\left(\frac{b(s)}{1+x^{n}(s-\tau(s))}\right)
$$

On the other hand,

$$
\begin{aligned}
(A x)(k) & =\sum_{s=k}^{k+\omega-1}\left(G(k, s) \frac{b(s)}{1+x^{n}(s-\tau(s))}\right) \\
& \geq m \sum_{s=k}^{k+\omega-1}\left(\frac{b(s)}{1+x^{n}(s-\tau(s))}\right) \\
& =m \sum_{s=0}^{\omega-1}\left(\frac{b(s)}{1+x^{n}(s-\tau(s))}\right) \geq m \frac{1}{M}\|A x\|=\delta\|A x\|
\end{aligned}
$$

which implies $A x \in \Omega$, hence we have $A \Omega \subset \Omega$.

LEMMA 2.8. Every solution $x(k)$ of equation (1.2) is positive.

Proof. Let $x(k)$ be the solution of equation (1.2), then we have

$$
x(k+1)-[1-a(k)] x(k)=\frac{b(k)}{1+x^{n}(k-\tau(k))} .
$$

Hence we get

$$
x(k)-[1-a(k-1)] x(k-1)=\frac{b(k-1)}{1+x^{n}(k-1-\tau(k-1))},
$$

$\left(\mathrm{E}_{2}\right) \quad x(k-1)-[1-a(k-2)] x(k-2)=\frac{b(k-2)}{1+x^{n}(k-2-\tau(k-2))}$,

$\left(\mathrm{E}_{3}\right) \quad x(k-2)-[1-a(k-3)] x(k-3)=\frac{b(k-3)}{1+x^{n}(k-3-\tau(k-3))}$,

$\left(\mathrm{E}_{k-1}\right)$

$$
\begin{aligned}
& x(2)-[1-a(1)] x(1)=\frac{b(1)}{1+x^{n}(1-\tau(1))}, \\
& x(1)-[1-a(0)] x(0)=\frac{b(0)}{1+x^{n}(0-\tau(0))} .
\end{aligned}
$$

Multiplying two sides of $\left(\mathrm{E}_{2}\right), \ldots,\left(\mathrm{E}_{k}\right)$ by $1-a(k-1),[1-a(k-1)][1-a(k-2)]$, $\ldots,[1-a(k-1)][1-a(k-2)] \ldots[1-a(2)],[1-a(k-1)][1-a(k-2)] \ldots[1-$ $a(2)][1-a(1)]$, respectively, we get

$\left(\mathrm{E}_{2}^{\prime}\right) \quad[1-a(k-1)] x(k-1)-[1-a(k-1)][1-a(k-2)] x(k-2)$

$$
=\frac{b(k-2)}{1+x^{n}(k-2-\tau(k-2))}[1-a(k-1)],
$$


$\left(\mathrm{E}_{3}^{\prime}\right) \quad[1-a(k-1)][1-a(k-2)] x(k-2)$

$$
\begin{aligned}
& -[1-a(k-1)][1-a(k-2)][1-a(k-3)] x(k-3) \\
& \quad=\frac{b(k-3)}{1+x^{n}(k-3-\tau(k-3))}[1-a(k-1)][1-a(k-2)],
\end{aligned}
$$

$$
\begin{aligned}
& \left(\mathrm{E}_{k-1}^{\prime}\right) \quad[1-a(k-1)][1-a(k-2)] \ldots[1-a(2)] x(2) \\
& -[1-a(k-1)][1-a(k-2)] \ldots[1-a(2)][1-a(1)] x(1) \\
& \quad=\frac{b(1)}{1+x^{n}(1-\tau(1))}[1-a(k-1)][1-a(k-2)] \ldots[1-a(2)],
\end{aligned}
$$

$\left(\mathrm{E}_{k}^{\prime}\right) \quad[1-a(k-1)][1-a(k-2)] \ldots[1-a(2)][1-a(1)] x(1)$

$$
\begin{aligned}
& -[1-a(k-1)][1-a(k-2)] \ldots[1-a(2)][1-a(1)][1-a(0)] x(0) \\
& =\frac{b(0)}{1+x^{n}(0-\tau(0))}[1-a(k-1)][1-a(k-2)] \ldots[1-a(2)][1-a(1)] .
\end{aligned}
$$

Summing $\left(\mathrm{E}_{1}\right),\left(\mathrm{E}_{2}^{\prime}\right),\left(\mathrm{E}_{3}^{\prime}\right), \ldots,\left(\mathrm{E}_{k-1}^{\prime}\right),\left(\mathrm{E}_{k}^{\prime}\right)$, we get

$$
\begin{aligned}
x(k) & -[1-a(k-1)][1-a(k-2)] \ldots[1-a(2)][1-a(1)][1-a(0)] x(0) \\
& =\frac{b(k-1)}{1+x^{n}(k-1-\tau(k-1))}+\sum_{s=0}^{k-2}\left(\frac{b(s)}{1+x^{n}(s-\tau(s))} \prod_{i=s+1}^{k-1}(1-a(i))\right) .
\end{aligned}
$$

That is

$$
\begin{aligned}
& x(k)=x(0) \prod_{s=0}^{k-1}(1-a(s))+\frac{b(k-1)}{1+x^{n}(k-1-\tau(k-1))} \\
&+\sum_{s=0}^{k-2}\left(\frac{b(s)}{1+x^{n}(s-\tau(s))} \prod_{i=s+1}^{k-1}(1-a(i))\right) .
\end{aligned}
$$

Since $x(k)=\phi(k)>0$ for $-\tau^{*} \leq k \leq 0$, we can deduce that $x(1)>0, \ldots$ $x(k)>0$ for all $k \in Z^{+}$.

Lemma 2.9. Every solution $x(k)$ of equation (1.2) is bounded.

Proof. Suppose that equation (1.2) has unbounded solution $x(k)$. Then, for sufficiently large $L>0$, there exists $K_{2} \in Z^{+}$and $x\left(K_{2}\right)$ sufficiently large, such that $-\underline{a} x\left(K_{2}\right)+\bar{b}<-L$. Thus we have

$$
\begin{aligned}
x\left(K_{2}+1\right)-x\left(K_{2}\right) & =-a\left(K_{2}\right) x\left(K_{2}\right)+\frac{b\left(K_{2}\right)}{1+x^{n}\left(K_{2}-\tau\left(K_{2}\right)\right)} \\
& \leq-a\left(K_{2}\right) x\left(K_{2}\right)+b\left(K_{2}\right) \leq-\underline{a} x\left(K_{2}\right)+\bar{b}<-L<0
\end{aligned}
$$


which implies $x\left(K_{2}+1\right)<x\left(K_{2}\right)$.

Let $\mu=x\left(K_{2}+1\right), K_{1}=\max \left\{k \mid x(k)<\mu, k<K_{2}\right\}$, then we have $x\left(K_{1}\right)<\mu$ and $x\left(K_{1}+1\right) \geq \mu$. Thus there exists $K_{3} \in Z^{+}$and $K_{1}+1 \leq K_{3} \leq K_{2}$, such that $x\left(K_{3}\right)=\max _{K_{1}+1 \leq k \leq K_{2}} x(k)$. Hence

$$
x\left(K_{3}-1\right) \leq x\left(K_{3}\right) .
$$

(2.3) can be divided into two cases:

Case 1. If $x\left(K_{2}\right)<x\left(K_{3}-1\right) \leq x\left(K_{3}\right)$, then

$$
\begin{aligned}
x\left(K_{3}\right)-x\left(K_{3}-1\right) & =-a\left(K_{3}-1\right) x\left(K_{3}-1\right)+\frac{b\left(K_{3}-1\right)}{1+x^{n}\left(K_{3}-1-\tau\left(K_{3}-1\right)\right)} \\
& \leq-\underline{a} x\left(K_{3}-1\right)+\bar{b}<-\underline{a} x\left(K_{2}\right)+\bar{b}<-L<0 .
\end{aligned}
$$

Thus $x\left(K_{3}\right)<x\left(K_{3}-1\right)$, which contradicts with (2.3).

Case 2. If $x\left(K_{3}-1\right) \leq x\left(K_{2}\right) \leq x\left(K_{3}\right)$, then

$$
\begin{aligned}
x\left(K_{3}\right)-x\left(K_{3}-1\right) & =-a\left(K_{3}-1\right) x\left(K_{3}-1\right)+\frac{b\left(K_{3}-1\right)}{1+x^{n}\left(K_{3}-1-\tau\left(K_{3}-1\right)\right)} \\
& \leq-\underline{a} x\left(K_{3}-1\right)+\bar{b}<-\underline{a} x\left(K_{3}-1\right)+\underline{a} x\left(K_{2}\right)-L \\
& \leq-\underline{a} x\left(K_{3}-1\right)+\underline{a} x\left(K_{3}\right)-L=\underline{a}\left[x\left(K_{3}\right)-x\left(K_{3}-1\right)\right]-L,
\end{aligned}
$$

Hence we get

$$
(1-\underline{a})\left[x\left(K_{3}\right)-x\left(K_{3}-1\right)\right]<-L,
$$

which yields

$$
x\left(K_{3}\right)-x\left(K_{3}-1\right)<-\frac{L}{1-\underline{a}}<0 .
$$

Thus $x\left(K_{3}\right)<x\left(K_{3}-1\right)$, which contradicts with (2.3). Therefore, every solution of equation (1.2) is bounded.

\section{Existence of the unique positive periodic solution}

Let $\widetilde{b}=\sum_{k=0}^{\omega-1} b(k)$, we make the following assumptions:

$\left(\mathrm{H}_{1}\right) \quad 0<n \leq 1$,

$\left(\mathrm{H}_{2}\right) n>1,(n-1)(M \widetilde{b})^{n} \leq 1$.

Theorem 3.1. Assume that $\left(\mathrm{H}_{1}\right)$ or $\left(\mathrm{H}_{2}\right)$ holds. Then equation (1.2) has a unique $\omega$-periodic positive solution $x^{*}(k)$. Moreover, $\left\|x_{k}-x^{*}\right\| \rightarrow 0,(k \rightarrow \infty)$, where $x_{k}=A x_{k-1}(k=1,2, \ldots)$ for any initial $x_{0} \in \Omega$.

Proof. Firstly, we prove that $\Omega$ is normal cone, $A: \Omega \rightarrow \Omega$ is decreasing operator.

For any $x, y \in \Omega,\|x\|=\|y\|=1$, we have $x(k) \geq 0, y(k) \geq 0, x(k) \geq \delta\|x\|=$ $\delta, y(k) \geq \delta\|y\|=\delta$, here $\delta=m / M$. Hence we get

$$
\|x+y\| \geq x(k)+y(k) \geq \delta+\delta=2 \delta .
$$


Thus there exists a positive constant $\sigma=2 \delta$, such that $\|x+y\| \geq \sigma$ for any $x, y \in \Omega,\|x\|=\|y\|=1$. So $\Omega$ is normal cone.

For $x, y \in \Omega, x \leq y$, then we have

$(A x)(k)-(A y)(k)=\sum_{s=k}^{k+\omega-1} G(k, s)\left(\frac{b(s)}{1+x^{n}(s-\tau(s))}-\frac{b(s)}{1+y^{n}(s-\tau(s))}\right) \geq 0$,

which implies $A x \geq A y$. So $A: \Omega \rightarrow \Omega$ is decreasing operator.

Now, we will show that condition (b) of Lemma 2.4 is satisfied.

$$
\begin{aligned}
M \widetilde{b} & =M \sum_{s=0}^{\omega-1} b(s)=M \sum_{s=k}^{k+\omega-1} b(s) \geq(A(0))(k) \\
& =\sum_{s=k}^{k+\omega-1}(G(k, s) b(s)) \geq m \sum_{s=k}^{k+\omega-1} b(s)=m \sum_{s=0}^{\omega-1} b(s)=m \widetilde{b}>0
\end{aligned}
$$

which implies $A(0)>0$. Again, we have

$$
\begin{aligned}
& \left(A^{2}(0)\right)(k)=\sum_{s=k}^{k+\omega-1}\left(G(k, s) \frac{b(s)}{1+(A(0))^{n}(s-\tau(s))}\right) \\
& \geq \frac{1}{1+(M \widetilde{b})^{n}} \sum_{s=k}^{k+\omega-1}(G(k, s) b(s))=\frac{1}{1+(M \widetilde{b})^{n}}(A(0))(k)=\varepsilon_{0}(A(0))(k) .
\end{aligned}
$$

This implies $A^{2}(0) \geq \varepsilon_{0} A(0)$, here $\varepsilon_{0}=1 /\left(1+(M \widetilde{b})^{n}\right)$.

Finally, we show that condition (c) of Lemma 2.4 is satisfied.

Let for any $0<a<c<1$, for any $a \leq \lambda \leq c$ and $0<x \leq A(0)$, we have $0<\|x\| \leq\|A(0)\| \leq M \widetilde{b}$.

$$
\begin{aligned}
A(\lambda x)(k) & =\sum_{s=k}^{k+\omega-1}\left(G(k, s) \frac{b(s)}{1+\lambda^{n} x^{n}(s-\tau(s))}\right) \\
& =\sum_{s=k}^{k+\omega-1}\left(G(k, s) \frac{b(s)}{1+x^{n}(s-\tau(s))} \cdot \frac{1+x^{n}(s-\tau(s))}{1+\lambda^{n} x^{n}(s-\tau(s))}\right) .
\end{aligned}
$$

Notice that

$$
\begin{aligned}
\frac{1+x^{n}(s-\tau(s))}{1+\lambda^{n} x^{n}(s-\tau(s))} & =\lambda^{-n}\left(1+\frac{\lambda^{n}-1}{1+\lambda^{n} x^{n}(s-\tau(s))}\right) \\
& \leq \lambda^{-n}\left(1+\frac{\lambda^{n}-1}{1+\lambda^{n}(M \widetilde{b})^{n}}\right)=\frac{1+(M \widetilde{b})^{n}}{1+\lambda^{n}(M \widetilde{b})^{n}}
\end{aligned}
$$

So we have

$$
\begin{aligned}
A(\lambda x)(k) & \leq \sum_{s=k}^{k+\omega-1}\left(G(k, s) \frac{b(s)}{1+x^{n}(s-\tau(s))} \cdot \frac{1+(M \widetilde{b})^{n}}{1+\lambda^{n}(M \widetilde{b})^{n}}\right) \\
& =\frac{1}{\lambda} \sum_{s=k}^{k+\omega-1}\left(G(k, s) \frac{b(s)}{1+x^{n}(s-\tau(s))} \cdot \frac{\lambda\left[1+(M \widetilde{b})^{n}\right]}{1+\lambda^{n}(M \widetilde{b})^{n}}\right) .
\end{aligned}
$$


Let

We have

$$
f(x)=\frac{\left[1+(M \widetilde{b})^{n}\right] x}{1+x^{n}(M \widetilde{b})^{n}} .
$$

$$
f^{\prime}(x)=\frac{\left[1+(M \widetilde{b})^{n}\right]\left[1+(1-n) x^{n}(M \widetilde{b})^{n}\right]}{\left[1+x^{n}(M \widetilde{b})^{n}\right]^{2}} .
$$

Since $\left(\mathrm{H}_{1}\right)$ or $\left(\mathrm{H}_{2}\right)$ holds, we know that $f^{\prime}(x)>0$ for $0<x<1$, so we have $0=f(0)<f(a) \leq f(\lambda) \leq f(c)<f(1)=1$. Hence we have

$$
\begin{aligned}
A(\lambda x)(k) & \leq \frac{1}{\lambda} \sum_{s=k}^{k+\omega-1}\left(G(k, s) \frac{b(s)}{1+x^{n}(s-\tau(s))} f(\lambda)\right) \\
& \leq \frac{1}{\lambda} \sum_{s=k}^{k+\omega-1}\left(G(k, s) \frac{b(s)}{1+x^{n}(s-\tau(s))} f(c)\right) \\
& =\frac{1}{\lambda} f(c) \sum_{s=k}^{k+\omega-1}\left(G(k, s) \frac{b(s)}{1+x^{n}(s-\tau(s))}\right) \\
& =\frac{1}{\lambda} f(c)(A x)(k)=\frac{1}{\lambda} \cdot \frac{1}{1+\eta(c)}(A x)(k),
\end{aligned}
$$

here $\eta=\eta(c)=1 / f(c)-1>0$.

By Lemma 2.4, we know operator $A$ has a unique positive fixed point $x^{*}>0$, $\left\|x_{k}-x^{*}\right\| \rightarrow 0,(k \rightarrow \infty), x_{k}=A x_{k-1}(k=1,2, \ldots)$ for any initial $x_{0} \in \Omega$.

REMARK 3.2. The Theorem 3.1 of this paper not only gives sufficient conditions for existence of unique positive periodic solution, but also gives iterative sequence $\left\{x_{k}\right\}$ which converges to $x^{*}$.

Remark 3.3. From the above proof, we have

$$
\begin{aligned}
x^{*}(k)=\left(A x^{*}\right)(k) & =\sum_{s=k}^{k+\omega-1}\left(G(k, s) \frac{b(s)}{1+x^{* n}(s-\tau(s))}\right) \\
& \leq M \sum_{s=k}^{k+\omega-1} b(s)=M \sum_{s=0}^{\omega-1} b(s)=M \widetilde{b} .
\end{aligned}
$$

We also have

$$
\begin{aligned}
x^{*}(k)=\left(A x^{*}\right)(k) & =\sum_{s=k}^{k+\omega-1}\left(G(k, s) \frac{b(s)}{1+x^{* n}(s-\tau(s))}\right) \\
& \geq m \sum_{s=k}^{k+\omega-1}\left(\frac{b(s)}{1+(M \widetilde{b})^{n}}\right)=\frac{m}{1+(M \widetilde{b})^{n}} \sum_{s=k}^{k+\omega-1} b(s) \\
& =\frac{m}{1+(M \widetilde{b})^{n}} \sum_{s=0}^{\omega-1} b(s)=\frac{m \widetilde{b}}{1+(M \widetilde{b})^{n}} .
\end{aligned}
$$

So we get $m \widetilde{b} /\left(1+(M \widetilde{b})^{n}\right) \leq x^{*}(k) \leq M \widetilde{b}$. 


\section{Global attractivity of positive periodic solution}

In this section, we study the global attractivity of positive periodic solution.

THEOREM 4.1. Assume that $\left(\mathrm{H}_{2}\right)$ holds, and $(n-1)^{n-1}(M \widetilde{b})^{n} \leq 1$, then equation (1.2) has a unique globally attractive $\omega$-periodic positive solution.

Proof. Since $\left(\mathrm{H}_{2}\right)$ holds, by Theorem 3.1 we know equation (1.2) has a unique $\omega$-periodic positive solution $x^{*}(k)$. Now we prove that $x^{*}(k)$ is globally attractive.

Suppose $x(k)$ is arbitrary solution of equation (1.2). Let $y(k)=x(k)-x^{*}(k)$, then we get

$$
\begin{aligned}
\Delta y(k) & =\Delta x(k)-\Delta x^{*}(k) \\
& =-a(k) y(k)+\frac{b(k)}{1+x^{* n}(k-\tau(k))}\left[\frac{1+x^{* n}(k-\tau(k))}{1+x^{n}(k-\tau(k))}-1\right] .
\end{aligned}
$$

Now we prove $\lim _{k \rightarrow+\infty} y(k)=0$ in three cases.

Case 1. Suppose $y(k)$ is eventually positive, then we have $y(k)=x(k)-$ $x^{*}(k)>0$ for $k$ sufficiently large. This combined with (4.1) leads to $\Delta y(k)<$ $-a(k) y(k)<0$. Thus we have $y(k+1)<y(k)$, which together with $y(k)>0$ implies $\lim _{k \rightarrow+\infty} y(k)=h \geq 0$.

We claim that $h=0$. If $h>0$, then we have $\Delta y(k)<-a(k) y(k)<-h a(k)$ for $k$ sufficiently large, i.e. there exists $N_{1}>0$, such that $\Delta y(k)<-h a(k)$ for all $k \geq N_{1}$.

Summing two sides of the inequality from $N_{1}$ to $+\infty$, we get

$$
h-y\left(N_{1}\right)=\sum_{k=N_{1}}^{+\infty} \Delta y(k)<-h \sum_{k=N_{1}}^{+\infty} a(k)=-\infty
$$

which is a contradiction, so we have $h=0$.

Case 2. Suppose $y(k)$ is eventually negative. By the similar proof as above, we also can get $h=0$.

Case 3. Suppose $y(k)$ is oscillatory. By Lemma 2.9, we know $x(k)$ and $x^{*}(k)$ are all bounded. So $y(k)$ is bounded.

Let $\lim _{k \rightarrow+\infty} \sup y(k)=p \geq 0, \lim _{k \rightarrow+\infty} \inf y(k)=q \leq 0$.

For for any $\varepsilon>0$ sufficiently small, there exists $N_{2}>0$, such that

$$
q-\varepsilon<y(k)<p+\varepsilon \quad \text { for all } k \geq N_{2} \text {. }
$$

From (4.1) we have

$$
y(k+1)-(1-a(k)) y(k)=\frac{b(k)}{1+x^{* n}(k-\tau(k))}\left[\frac{1+x^{* n}(k-\tau(k))}{1+x^{n}(k-\tau(k))}-1\right] .
$$


Multiplying the two sides by $\prod_{s=0}^{k} 1 /(1-a(s))$, we get

$$
\begin{aligned}
& \Delta\left(y(k) \prod_{s=0}^{k-1} \frac{1}{1-a(s)}\right) \\
& =\frac{b(k)}{1+x^{* n}(k-\tau(k))}\left[\frac{1+x^{* n}(k-\tau(k))}{1+x^{n}(k-\tau(k))}-1\right] \prod_{s=0}^{k} \frac{1}{1-a(s)} \\
& =\frac{b(k)}{1+x^{* n}(k-\tau(k))}\left[\exp \left(\ln \left[\frac{1+x^{* n}(k-\tau(k))}{1+x^{n}(k-\tau(k))}\right]\right)-1\right] \prod_{s=0}^{k} \frac{1}{1-a(s)} .
\end{aligned}
$$

By mean value theorem, we have

$$
\begin{aligned}
\ln & {\left[\frac{1+x^{* n}(k-\tau(k))}{1+x^{n}(k-\tau(k))}\right] } \\
& =\ln \left(1+x^{* n}(k-\tau(k))\right)-\ln \left(1+x^{n}(k-\tau(k))\right) \\
& =\frac{n \xi^{n-1}}{1+\xi^{n}}\left(x^{*}(k-\tau(k))-x(k-\tau(k))\right)=-\frac{n \xi^{n-1}}{1+\xi^{n}} y(k-\tau(k))
\end{aligned}
$$

where $\xi$ lies between $x^{*}(k-\tau(k))$ and $x(k-\tau(k))$.

Notice that the function $g(x)=n x^{n-1} /\left(1+x^{n}\right)$ is increasing on $[0, \sqrt[n]{n-1}]$ and decreasing on $[\sqrt[n]{n-1},+\infty)$. So $g(x)=n x^{n-1} /\left(1+x^{n}\right)$ has maximum $(n-1)^{1-1 / n}$ on $[0,+\infty)$. Hence we have $n \xi^{n-1} /\left(1+\xi^{n}\right) \leq(n-1)^{1-1 / n}$, which together with (4.2) and (4.4) yields

$$
\ln \left[\frac{1+x^{* n}(k-\tau(k))}{1+x^{n}(k-\tau(k))}\right] \leq-(q-\varepsilon)(n-1)^{1-1 / n},
$$

in which $q-\varepsilon<0$. Thus, by (4.3) and (4.5), we get

$$
\begin{aligned}
& \Delta\left(y(k) \prod_{s=0}^{k-1} \frac{1}{1-a(s)}\right) \\
& \quad \leq\left[\exp \left(-(q-\varepsilon)(n-1)^{1-1 / n}\right)-1\right] \frac{b(k)}{1+x^{* n}(k-\tau(k))} \prod_{s=0}^{k} \frac{1}{1-a(s)} \\
& \quad=\left[\exp \left(-(q-\varepsilon)(n-1)^{1-1 / n}\right)-1\right] \Delta\left(x^{*}(k) \prod_{s=0}^{k-1} \frac{1}{1-a(s)}\right),
\end{aligned}
$$

Summing two sides of (4.6) from $N_{2}$ to $k,\left(k>N_{2}\right)$, we get

$$
\begin{aligned}
y(k+1) \prod_{s=0}^{k} \frac{1}{1-a(s)}- & y\left(N_{2}\right) \prod_{s=0}^{N_{2}-1} \frac{1}{1-a(s)} \\
\leq & {\left[\exp \left(-(q-\varepsilon)(n-1)^{1-1 / n}\right)-1\right] } \\
& \cdot\left(x^{*}(k+1) \prod_{s=0}^{k} \frac{1}{1-a(s)}-x^{*}\left(N_{2}\right) \prod_{s=0}^{N_{2}-1} \frac{1}{1-a(s)}\right) .
\end{aligned}
$$


This leads to

$$
\begin{aligned}
y(k+1) \leq & y\left(N_{2}\right) \prod_{s=N_{2}}^{k}(1-a(s))+\left[\exp \left(-(q-\varepsilon)(n-1)^{1-1 / n}\right)-1\right] \\
& \cdot\left(x^{*}(k+1)-x^{*}\left(N_{2}\right) \prod_{s=N_{2}}^{k}(1-a(s))\right) \\
\leq & y\left(N_{2}\right) \prod_{s=N_{2}}^{k}(1-a(s))+\left[\exp \left(-(q-\varepsilon)(n-1)^{1-1 / n}\right)-1\right] \\
& \cdot\left(M \widetilde{b}-x^{*}\left(N_{2}\right) \prod_{s=N_{2}}^{k}(1-a(s))\right) .
\end{aligned}
$$

Let $k \rightarrow+\infty$, we get $p \leq M \widetilde{b}\left[\exp \left(-(q-\varepsilon)(n-1)^{1-1 / n}\right)-1\right]$. As $\varepsilon$ is arbitrary, we have

$$
p \leq M \widetilde{b}\left[\exp \left(-q(n-1)^{1-1 / n}\right)-1\right] .
$$

Now we prove that the inequality $q \geq M \widetilde{b}\left[\exp \left(-p(n-1)^{1-1 / n}\right)-1\right]$ holds too. By similar method as above, from $n \xi^{n-1} /\left(1+\xi^{n}\right) \leq(n-1)^{1-1 / n}$ and (4.2), (4.4), we get

$$
\ln \left[\frac{1+x^{* n}(k-\tau(k))}{1+x^{n}(k-\tau(k))}\right] \geq-(p+\varepsilon)(n-1)^{1-1 / n},
$$

Hence, by (4.3) and (4.8), we obtain

$$
\begin{aligned}
& \Delta\left(y(k) \prod_{s=0}^{k-1} \frac{1}{1-a(s)}\right) \\
& \geq\left[\exp \left(-(p+\varepsilon)(n-1)^{1-1 / n}\right)-1\right] \frac{b(k)}{1+x^{* n}(k-\tau(k))} \prod_{s=0}^{k} \frac{1}{1-a(s)} \\
& =\left[\exp \left(-(p+\varepsilon)(n-1)^{1-1 / n}\right)-1\right] \Delta\left(x^{*}(k) \prod_{s=0}^{k-1} \frac{1}{1-a(s)}\right) .
\end{aligned}
$$

Summing two sides of (4.9) from $N_{2}$ to $k,\left(k>N_{2}\right)$, we get

$$
\begin{aligned}
y(k+1) \prod_{s=0}^{k} \frac{1}{1-a(s)}- & y\left(N_{2}\right) \prod_{s=0}^{N_{2}-1} \frac{1}{1-a(s)} \\
\geq & {\left[\exp \left(-(p+\varepsilon)(n-1)^{1-1 / n}\right)-1\right] } \\
& \cdot\left(x^{*}(k+1) \prod_{s=0}^{k} \frac{1}{1-a(s)}-x^{*}\left(N_{2}\right) \prod_{s=0}^{N_{2}-1} \frac{1}{1-a(s)}\right) .
\end{aligned}
$$


This yields

$$
\begin{aligned}
y(k+1) \geq & y\left(N_{2}\right) \prod_{s=N_{2}}^{k}(1-a(s))+\left[\exp \left(-(p+\varepsilon)(n-1)^{1-1 / n}\right)-1\right] \\
& \cdot\left(x^{*}(k+1)-x^{*}\left(N_{2}\right) \prod_{s=N_{2}}^{k}(1-a(s))\right) \\
\geq & y\left(N_{2}\right) \prod_{s=N_{2}}^{k}(1-a(s))+\left[\exp \left(-(p+\varepsilon)(n-1)^{1-1 / n}\right)-1\right] \\
& \cdot\left(M \widetilde{b}-x^{*}\left(N_{2}\right) \prod_{s=N_{2}}^{k}(1-a(s))\right) .
\end{aligned}
$$

Let $k \rightarrow+\infty$. We get $q \geq M \widetilde{b}\left[\exp \left(-(p+\varepsilon)(n-1)^{1-1 / n}\right)-1\right]$. As $\varepsilon$ is arbitrary, we have

$$
q \geq M \widetilde{b}\left[\exp \left(-p(n-1)^{1-1 / n}\right)-1\right] .
$$

From the condition $(n-1)^{n-1}(M \widetilde{b})^{n} \leq 1$, we have $M \widetilde{b}(n-1)^{1-1 / n} \leq 1$. Since $p \geq 0, q \leq 0$, by $M \widetilde{b}(n-1)^{1-1 / n} \leq 1$ and the results in [18], we know that (4.7) and (4.10) have a unique solution $p=q=0$. So we get $\lim _{k \rightarrow+\infty} y(k)=0$. This implies $x^{*}(k)$ is globally attractive.

REMARK 4.2. By means of the well-known inequality $x^{x} \geq x$ for $x>0$, we get $(n-1)^{n-1} \geq n-1$ for $n>1$. Hence the inequality $(n-1)^{n-1}(M \widetilde{b})^{n} \leq 1$ implies $(n-1)(M \widetilde{b})^{n} \leq 1$. Thus the condition of Theorem 4.1 can be replaced by $n>1$ and $(n-1)^{n-1}(M \widetilde{b})^{n} \leq 1$.

So we have the following theorem.

Theorem 4.3. Assume that $n>1$ and $(n-1)^{n-1}(M \widetilde{b})^{n} \leq 1$, then equation (1.2) has a unique globally attractive $\omega$-periodic positive solution.

Acknowledgements. The author thanks the referees for their valuable comments and suggestions in improving the presentation of the manuscript.

\section{REFERENCES}

[1] R.P. Agarwal, Difference Equations and Inequalities: Theory, Methods and Applications, 2nd ed., Monographs and Textbooks in Pure and Applied Mathematics, vol. 228, Marcel Dekker, New York, 2000.

[2] A. Anguraj, M.M. Arjunan and H.M.Eduardo, Existence results for an impulsive neutral functional differential equation with state-dependent delay, Appl. Anal.86 (7) (2007), 861-872.

[3] M.M.A. Elsheikh, A. Zaghrout And A. Ammar, Oscillation and global attractivity in delay equation of population dynamics, Appl. Math. Comput. 77 (1996), 195-204. 
[4] M. FAN AND K. WANG, Global existence of positive periodic solutions of periodic predatorprey system with infinite delays, J. Math. Anal. Appl. 262 (2001), 1-11.

[5] K. Gopalsamy, M.R.S. Kulenovic and G.Ladas, Oscillation and global attractivity in models of Hematopoiesis, J. Dynam. Differential Equations 2 (1990), 117-132.

[6] D. Guo, Nonlinear Functional Analysis, Shandong Science and Technology Press, Jinan, 2001.

[7] I. Gyori And G. Ladas, Oscillation Theory of Delay Differential Equations with Applications, Clarendon Press, Oxford, 1991.

[8] H.F. Huo, W.T. Li AND X.Z. LiU, Existence and global attractivity of positive periodic solution of an impulsive delay differential equation, Appl. Anal. 83 (12) (2004), 1279-1290.

[9] D.Q. JIANG, Existence of positive periodic solutions for non-autonomous delay differential equations, Chinese Ann. of Math. 20A (6) (1999), 715-720.

[10] G. Karakostas, C.G. Philos and Y.G. Sficas, Stable steady state of some population models, J. Dynam. Differential Equations 4 (1992), 161-190.

[11] Y. KuAng, Delay Differential Equations with Applications in Population Dynamics, Academic Press, New York, 1993.

[12] W.T. Li AND H.F. HuO, Existence and global attractivity of positive periodic solutions of functional differential equations with impulses, Nonlinear Anal. 59 (6) (2004), 857-877.

[13] M.J. MA AND J.S. YU, Existence of multiple positive periodic solutions for nonlinear functional difference equations, J. Math. Anal. Appl. 305 (2002), 483-490.

[14] M.C. MaCkey AND L. GLASS, Oscillations and chaos in physiological control systems, Sciences 197 (1977), 287-289.

[15] S.H. SAKER, Oscillation and global attractivity in Hematopoiesis model with time delay, Appl. Math. Comput. 136 (2003), 241-250.

[16] _ Oscillation and global attractivity in Hematopoiesis model with periodic coefficients, Appl. Math. Comput. 142 (2003), 477-494.

[17] A. Wan, D. JiAng And X. XU, A new existence theory for positive periodic solutions to functional differential equations, Computers Math. Appl. 47 (2004), 1257-1262.

[18] W. XU AND J. LI, Global attractivity of the model for the survival of red blood cells with several delays, Ann. Differential Equations 14 (2) (1998), 357-363.

[19] D. Ye And M. FAn, Periodicity in mutualism systems with impulse, Taiwanese J. Math. 10 (3) (2006), 723-737.

\section{ZHIJIAN YAO}

Department of Mathematics and Physics

Anhui University of Architecture

Hefei, Anhui, 230601, P.R. CHINA

E-mail address: zhijianyao@126.com 